\title{
AOR
}

Selected Papers of \#AolR2020:

The $21^{\text {st }}$ Annual Conference of the

Association of Internet Researchers

Virtual Event / 27-31 October 2020

\section{MEDIATING PRECARITY THROUGH MOBILE APPS}

Ragan Glover-Rijkse

North Carolina State University

Melissa Stone

North Carolina State University

Megan Fletcher

North Carolina State University

Gayas Eapen

North Carolina State University

\section{Panel Rationale}

Precarity is a loaded term, but one that often connotes insecurity, risks, and threats to life itself. According to Mullenbach (2013), "precarity" operates as "shorthand for those of us documenting the multiple forms of nightmarish dispossession and injury that our age entails" (p. 298). Precarity, therefore, does not refer to any one experience, but rather a "rubric that brings together women, queers, transgender people, the poor, and the stateless" under a common life experience (Butler, 2009, p. xiii). Increasingly, we have witnessed an uptick in populations uniting under this common experience through the use of internet-connected mobile applications. These apps allow precarious populations to connect with others in real-time, to ascertain information about current risks, and to find a community in nearby spaces. In short, they allow these populations to mediate their precarity. Yet, as internet studies has taught us, online spaces rarely if ever offer seamless and friction-free encounters, even in spaces carved out specifically for marginalized populations (Gray, 2012; Shaw, 2014). In this panel, we take up this perspective to problematize the recent use of mobile apps for mediating experiences of precarity. While we acknowledge that these apps offer opportunities to form networks of resistance, these apps also pose substantial risks to their users and their livelihood. To begin to articulate these risks, the presentations in this panel consider case studies of these apps, targeted at three different experiences of precarity.

Suggested Citation (APA): Glover-Rijkse, R., Stone, M., Fletcher, M., Eapen, G. (2020, October).

Mediating Precarity Through Mobile Apps. Panel Presented at Aoir 2020: The $21^{\text {th }}$ Annual Conference of the Association of Internet Researchers. Virtual Event: AolR. Retrieved from http://spir.aoir.org. 
First, we consider the precarity that arises as different identities move through urban spaces, encountering unknown others who might pose a threat. These apps, framed as "safety-oriented mobility" apps, allow users to track and report location-specific instances of harassment and violence. We consider how these apps are situated as an intervention to precarious urban mobilities, allowing users to avoid dangerous spaces; however, we also complicate this claim to suggest that these apps can reinforce harmful homogeneity in spaces, enable surveillance of marginalized populations, and provide a false sense of security to users. Moving beyond urban spaces, we recognize that more often than not, acts of harassment and violence occur between people who already know one another in the private space of the home. As such, in this next presentation, we examine apps that have responded to intimate partner violence. We discuss the possibilities for these apps to help women connect with a network, track instances of violence, and exit abusive relationships. Nevertheless, abusers can also weaponize these apps to enact forms of surveillance and control. We argue that ultimately these mobile apps do very little to protect their users from harm and, instead, provide a shortterm distraction from underlying issues. In a final move, we shift from apps that help users to avoid (harmful) people in the physical world to consider mobile apps that help users to connect with a nearby network of people with a similar identity. However, these nearby networks are not free from the risks of the physical world. In this presentation, we look specifically to LBGTQ+ apps aimed at finding romantic partners or coordinating sexual encounters through location sharing. While these apps purport to mediate the precarity of performing sexual identity in public places, recent upticks in data breaches and leaks increasingly pose a risk of unwanted exposure to the LBGTQ+ individuals who use these apps. As such, we argue for these apps to make their mediation and associated risks more evident to users.

Collectively, the presentations in this panel demonstrate how mobile apps may amplify, rather than mollify, experiences of precarity. By way of conclusion, we offer a collective statement which argues for systemic intervention addressing the inequalities within society but, until that time comes, we argue for measures that secure these mobile apps (and the data contained therein) and protect their users. Such an intervention is critical in our current conjuncture, as we recognize the inextricable intertwinement of our physically and digitally networked lives.

\section{References}

Butler, J. (2009). Performativity, Precarity and Sexual Politics. AIBR. Revista de Antropología Iberoamericana, 04(03), I-XIII. https://doi.org/10.11156/aibr.040303e 
Gray, K. L. (2012). Intersecting oppressions and online communities: Examining the experiences of women of color in Xbox Live. Information, Communication \& Society, 15(3), 411-428.

Muehlebach, A. (2013), On Precariousness and the ethical imagination: The Year 2012 in Sociocultural Anthropology. American Anthropologist, 115: 297-311. doi:10.1111/aman.12011

Shaw, A. (2014). The internet is full of jerks, because the world is full of jerks: What feminist theory teaches us about the internet." Communication and critical/cultural Studies, 11(3), pp. 273-277. 
Panel Abstracts

\section{MOBILE SAFETY APPS: A MATERIALIST FEMINIST ORIENTATION TO PRECARIOUS MOBILITIES}

Ragan Glover-Rijkse

North Carolina State University

Melissa Stone

North Carolina State University

In recent years, a number of safety-oriented mobile apps have allowed users to report location-specific instances of public harassment and violence. These apps encourage the development of "networks of resistance" against such acts (Hart \& Michael, 2015); and they engage storytelling as means to "exercise agency, shape identity, and motivate action" (Dimond, Dye, LaRose, \& Bruckman, 2013, p. 477). Importantly, though, many of these apps also offer digital cartographies, reflecting spatial narratives of harassment and violence. These digital cartographies enable the tech-savvy and mobile phone equipped user to move through urban spaces, reading the urban landscape for safe paths and writing the urban landscape with their experiences of mobility. While practices of "reading and writing space" are often associated with playful, creative, and experimental encounters with urban space (de Souza e Silva \& Frith, 2012), this presentation contends that some users engage these practices as a necessity for self- and community- preservation. While it may be tempting to position mobile apps as yet another platform through which particular communities interact, coordinate activity, and/or share resources, doing so would elide the particular affordance that mobile apps offer for forming an always-on, networked co-presence with a community of users. From this perspective, mobile devices become an interface for contending with precarious mobilities in urban spaces and generating a sense of safety through numbers.

This presentation therefore examines several of these safety-oriented mobile apps (e.g. Hollaback!, Islamophobia, Prejudice Tracker, Safety Net) to consider how concerns for self-preservation might shape mobilities in urban spaces. For instance, the mobile app Safety Net allows sex workers to anonymously report instances of sexual violence via the app and to immediately broadcast a warning notification to other sex workers within the area, detailing the incident and where it occurred. Similarly, the mobile app Islamaphobia allows users to report locations and descriptions of Islamophobia and to traverse other users' reports. The app operates under the tagline 'if you don't report it, it never happened" and has an accompanying explanation which indicates that changes 
in public policy will only occur by producing a record of Islamophic incidents. Together, these apps serve two functions for users: to protect and be protected. Put differently, safety-oriented mobile apps situate users within a community, instilling in these users an ethical imperative to preserve and protect that community. Moreover, these apps also offer users the ability to interact with digitally crowdsourced information to inform their own safety-oriented mobilities.

To arrive at such analysis, this presentation draws from critical mobilities studies and feminist new materialist approaches to understand the complex relations that produce mobilities. A critical mobilities perspective offers a useful approach to examining how the interplay between the technical and social field converge to construct mobility/immobility as well as the experience of it. It also attends to aspects of identity, power relations, and inequality that shape mobilities. Complementing critical mobilities, a feminist new materialist approach offers insight into multiple agentic relations that cohere to configure spaces/places-importantly, though, a feminist new materialist approach understands space/place as ontologically unstable. Safety-oriented mobility apps, therefore, respond to this instability, while simultaneously becoming constituted within these relations. This approach is informed by McAlister and Ewalt (2018) who suggest that these two approaches "prompt radical reimaginations of place-based politics and spatial agencies by encouraging attention to animate matter, natural-cultural assemblages, and new forms of safety and precarity that follow from the differential distribution of movement" (p. 339). This presentation therefore avoids suggesting that mediated mobilities are necessarily agentic. Instead, it considers the power relations that emerge through ongoing negotiations of identity, space/place, (im)mobility, and networked digital devices. To include matters of power and safety, this presentation "fine-tunes [its] accounts of the politics of mobility" (Cresswell, 2010, p. 17) and treats socially constructed identities as a "question that inflects power relations throughout all contexts" (Shaw, 2014, p. 273).

This presentation argues that while safety-oriented mobile apps offer marginalized populations the chance to avoid potentially problematic places by navigating to/through safe urban spaces, these technologies should be met with a degree of skepticism, which acknowledges their potential for creating harmful homogeneity and continuing hegemonic surveillance. Indeed, some anecdotes suggest that these apps have been used to harmfully target and track the apps' intended users. Thus, despite the intention for these apps to produce safety-oriented mobilities, they may, in fact, pose new risks. Moreover, these apps may provide a false sense of safety, as "networked connectivity" gets conflated with "safety." For example, in the past year, a college student in the southeastern United States was murdered by an Uber driver, while her boyfriend tracked her location on his phone, aware that something was wrong, but powerless to 
do anything about the situation. As such, these apps draw attention to the limitations of networked co-presence for producing meaningful intervention.

By analyzing the affordances and limitations of safety-oriented mobile apps, the definition of safety and how it is achieved becomes refigured. This presentation complicates the belief that carrying a mobile device and sharing location-information with a network necessarily produces safety. Rather, safety becomes bound up in what it means to move through destabilized spaces - it implies attending to flux as relations constantly change and negotiating precarity as a mode of being. Safety-oriented mobile apps attune users to this state of flux, while simultaneously becoming imbricated in it.

\section{References}

Cresswell, T. (2016). Towards a politics of mobility. In Routes, Roads and Landscapes (pp. 181-196). Routledge Press.

de Souza e Silva, A., \& Frith, J. (2014). Re-narrating the city through the presentation of location.In J. Farman (Ed.), The mobile story: Narrative practices with locative technologies (pp. 34-49). New York, NY: Routledge.

Dimond, J. P., Dye, M., Larose, D., \& Bruckman, A. S. (2013). Hollaback!: The role of storytelling online in a social movement organization. Proceedings of the 2013 Conference on Computer Supported Cooperative Work - CSCW '13, 477. https://doi.org/10.1145/2441776.2441831

Hart, L., \& Mitchell, C. (n.d.). From spaces of sexual violence to sites of networked resistance: Re-imagining mobile and social media technologies. Perspectives in Education, 17.

McAlister, J. F., \& Ewalt, J. P. (2018). New Materialities and Precarious Mobilities: Reinventing Studies of Space and Place. Women's Studies in Communication, 41(4), 339-348. https://doi.org/10.1080/07491409.2018.1551680

Shaw, A. (2014). The internet is full of jerks, because the world is full of jerks: What feminist theory teaches us about the internet." Communication and critical/cultural Studies, 11(3), pp. 273-277.

\section{PROTECTION IN THE PALM OF YOUR HAND? THE PRECARITY OF PERSONAL SAFETY APPS FOR WOMEN}


Megan Fletcher

North Carolina State University

"Text me when you get home." This commonly used parting phrase encapsulates the social understanding that a woman's safety is at risk when she is alone. Most of the work that goes into policing and lessening that risk falls on women's ability to adhere to socially constructed rules: walk in groups, do not travel alone at night, stick to well-lit areas and highly trafficked roads or paths. More recently, these tropes have taken a digital turn. Mobile applications concerned with personal safety, with a primary focus on the protection and safety of women are on the rise (Hart \& Mitchell, 2015). Surveillance based applications are among the most common which include popular mobile applications like Companion and bSafe which have been downloaded over 1 million times. Now, there are a variety of mobile applications aimed towards helping victims of Intimate Partner Violence (IPV). These applications include tools to evaluate the safety of relationships (OneLove My Plan, iMatter), send text messages to trusted contacts in dangerous situations (Early Rescue, Aspire News App, Circle of 6), and document instances of abuse (Aspire News App, Bright Sky).

This presentation situates itself within scholarly discourse regarding violence, gender, and communication networks. It engages the Circle of 6 as a locative mobile social network (de Souza e Silva \& Frith, 2012) concerned with soliciting a web of safety via social connections (Evans \& Feder, 2016; Hayes, 2013) and in doing so illuminates the precarity of personal safety apps for women. "Protection in the Palm Of Your Hand" speaks to a recent shift towards using digital technology as a resource for victims to report and exit abusive relationships. This presentation interrogates the conceptualization of the Circle of 6 mobile application and its functions, focusing primarily on its relevance to intimate partner violence. The National Coalition Against Domestic Violence (NCADV) claims that intimate partner violence can affect anyone, however it is most often perpetrated by men against women, which makes intimate partner violence a gendered issue. According to Sharma (2017) women's agency in entering and exiting space is historically disenfranchised. This becomes even more problematic when considering the inherent power imbalance that characterizes intimate partner violence. Intimate Partner Violence is characterized by surveillance practices; thus resources for victims of abuse have historically been discreet in an effort to avoid attention from abusers, which if recognized could further threaten their safety (Woodlock, 2017; Evans \& Feder, 2016; Hayes, 2013). Mason and Magnet (2012) examine the ways in which technological surveillance has been weaponized against women in abusive relationships; however, they also note how that same technology is now being recognized as a means of resistance and protection for women against abuse. 
The Circle of 6 mobile application relies extensively on the pre-existing social networks of victims and access to mobile technology in order to facilitate networked support through reporting and intervention, which could ultimately allow for the victim to exit the abusive relationship. However, previous research regarding intimate partner violence and communication networks suggest a variety of difficulties victims face when trying to establish and maintain such networks (Mason \& Magnet, 2012; Goodboy \& Bolkan, 2011). Additionally, resources and network-making power can be sabotaged by abusers, leaving victims unable to generate or summon a network of support. The Circle of 6 app attempts to provide victims with a sense of protection regarding abuse and assault. However, it ultimately overlooks the historical and contextual forces within abusive relationships. Abusive relationships are often marked by control over women's bodies, social networks, and mobility. While the Circle of 6 application is framed as a resource for victims of intimate partner violence, further analysis complicates whether it is capable of serving as a method of resistance, ultimately empowering victims' access to exit

For optimal function "Tech 4 Good", the Circle of 6 development team, suggests picking reliable friends who are likely to respond to an impromptu text. This suggestion proves especially problematic when considering who the mobile application's target audience is. Research suggests that women in abusive relationships have a difficult time maintaining social connections and use anti-social strategies as a way to protect and sustain the abusive relationship (Goodboy \& Bolkan, 2011). The app's developers emphasize choosing contacts who understand the responsibilities of being a part of the "circle," and suggest that users choose contacts who live within close proximity and are able to travel to the specified location (Tech 4 Good, 2015). According to McAlister and Ewalt (2018) mobility studies considers how "human bodies, digital information, capital, and discourses - moving at different speeds and with different degrees of freedomgenerate varying safeties and precarities within a broader set of sociospatial relations" (p. 342). Variables are subject to change at any moment, yet personal safety apps prime women to see themselves as being in control of their safety (Gill, 2008).

The Circle of 6 and other personal safety apps are partially paradoxical. The development and continued existence of personal safety apps acknowledges the very real crisis that sexual assault, rape and intimate partner violence pose for women-yet the solutions they offer perpetuate problematic and unsubstantial responses to violence against women and ignore the means of mobility and immobility that characterize and shape communicative practices within intimate partner violence (McAlister \& Ewalt, 2018). This presentation hopes to illuminate these issues in an effort to catalyze systemic interventions that recognize these complexities. 


\section{References}

de Souza e Silva, A. \& Frith, J. (2012). Mobile interfaces in public spaces: Locational privacy, control, and urban sociability. Routledge.

Evans, M. A., \& Feder, G. S. (2016). Help-seeking amongst women survivors of domestic violence: A qualitative study of pathways towards formal and informal support. Health Expectations, 19(1), 62-73. 10.1111/hex.12330

Gill, R. (2008). Culture and subjectivity in neoliberal and postfeminist times. Subjectivity, 25(1), 432-445.

Goodboy, A. K., \& Bolkan, S. (2011). Attachment and the use of negative relational maintenance behaviors in romantic relationships. Communication Research Reports, 28(4), 327-336. doi:10.1080/08824096.2011.616244

Hart, L., \& Mitchell, C. (2015). From spaces of sexual violence to sites of networked resistance: Re-imagining mobile and social media technologies. Perspectives in Education, 33(4), 135-150.

Hayes, B. E. (2013). Women's resistance strategies in abusive relationships. SAGE Open, 3(3), 215824401350115. 10.1177/2158244013501154

Mason, C. \& Magnet, S. (2012). Surveillance studies and violence against women. Surveillance \& Society, 10(2), 105.

McAlister, J. F., \& Ewalt, J. P. (2018). New Materialities and Precarious Mobilities: Reinventing Studies of Space and Place. Women's Studies in Communication, 41(4), 339-348. https://doi.org/10.1080/07491409.2018.1551680

Sharma, S. (2017). Exit and the Extensions of Man. Transmediale Online Journal, April. Tech 4 Good (2015). Circle of 6 . Retrieved February 27, 2020, from Circle of 6 website: https://www.circleof6app.com:443/

Woodlock, D. (2017). The abuse of technology in domestic violence and stalking. Violence Against Women, 23(5), 584-602. 10.1177/1077801216646277 


\section{BREACHES/LEAKS IN ‘QUEER’ APPS: LOCATION DATA, PLACEMAKING, AND SPATIAL RECONFIGURATION}

\section{Gayas Eapen}

North Carolina State University

Reports of data breaches, leaks and security risks in mobile applications - especially dating applications - have become common in data security portals. For instance, in August 2019 ZDnet.com reported that on four dating applications, users' personal information was easily accessible. These apps included Grindr (LBGTQ+ dating app), 3Fun (a dating app for arranging threesomes) as well as Romeo and Recon (apps targeted at gay men). The main emphasis of this report was the vulnerability of location data, which could be made available using computational processes, such as triangulation and trilateration, and plotted onto maps to analyze patterns of use. This presentation examines these data leaks and breaches to understand how a user's location is closely tied to placemaking practices-contingent on a place's social relations and the embodiment of people within them. Aware that not all people have equitable access to participate in place/space(s), this presentation explores the control exerted by 'places' in disciplining non-normative sexualities. It also considers user practices, like location spoofing, which seek to mitigate this control.

In particular, this presentation analyzes the data breaches in "queer" dating apps, 3Fun and Grindr. To offer this analysis, this presentation offers a close reading of reports of these data breaches. Close reading reveals how these apps function in the public domain and offers insights into how users change their everyday behaviour when they encounter these applications' vulnerabilities based on such reporting. It draws from the understanding that queer apps proliferate, leak, and insinuate themselves in the everyday lives of people (Shah, 2012). Yet it additionally recognizes that the internet's infrastructures, constituted through the homophobic, capitalist state, are in constant opposition to queer, indeterminate and ambivalent experiences (Cockayne \& Richardson, 2019). As such, this indeterminacy and ambivalence get mirrored in digital mediations and are identified by the fear of being made visible, outed and ousted, and perceived as dysfunctional.

Anna McCarthy (2001) emphasises the everydayness of places in discussing modern mobile subjects. In extending McCarthy's work, this presentation focuses on sites where media objects are located to contextualize the embodied use of dating applications. Furthermore, this presentation attends to how power and control operate in spatial and relational terms. Through a close reading of breaches and leak reports, this presentation complicates the idea that location says something about identity (de Souza 
e Silva \& Frith, 2012) as these apps conflate multiple identities and distort location as the place of use. For instance, in one 2015 data leak, the pastor of a Baptist church in New Orleans was revealed to be using a dating service for extra-marital affairs. The disciplining practices of the church, along with the identity of the user as a church official, are tied together along with that of a queer subject. Similarly, in a 2019 breach of the 3Fun app, the existence of users in the US Supreme Court and the White House generated immense surprise because of the dissonance between the act and the associations of the place. However, the reports analyzed for this presentation suspect that some of these users were spoofing their locations to be present in these places.

Location spoofing - a practice of morphing or falsifying one's location in dating applications- has complicated the questions of 'proximity' or "site specificity" of action. Spoofing has become a way for users to protect themselves from the vulnerabilities of large-scale data collection and subsequent exposure. Leaks and breaches give a window into understanding the extent of data collection on these apps and how certain users attempt to mitigate sensemaking processes of these data. Subversive practices such as spoofing attempt to resist data-driven urban spatial reconstitution, where a spatial sorting of bodies is made possible through the use of vast personal data and algorithmic processes. In this context, I read spoofing as an escape from the tyranny of historically heteronormative place(s) and systems.

The existing work on location-based apps has already focused on the hybridization of the physical and the virtual (Miles, 2017) as well as the co-constituting and producing of bodies and spaces using mobile technologies (Farman, 2012). Nash and Murray (2019) similarly discuss placemaking practices - that draw on the mobilities paradigm - to contextualize unequal participation of subjects based on power dynamics in social relations as well places where these subjects are (non-)operational. Further, Shamas (2020) uses the idea of data-bodies, instead of datasets, to "draw explicit connections to the bodies in which we navigate the world." This presentation expands on these ideas, using the cases of leaks and breaches in queer apps to understand how location data can also be potentially employed in spatial sorting/reconfiguring of bodies. While perhaps contingent on the ideology of the people developing and employing apps, algorithmic spatial reconfiguration has already been observed in neighbourhood segregation based on race and income (Safransky, 2019). There is no guarantee that we will not see such discriminatory practices perpetuated through apps. This fear was particularly prominent when the data of HIV+ people were leaked from Grindr in 2019.

By analyzing the leaks and breaches in queer dating apps, this presentation demonstrates how the operations of black-boxed technological processes (apps in this case) can be made visible and analyzed, especially bringing together the 'technical' 
(location) and cultural-historical (place). This reflects a shift from looking at technological mediation of sexuality as simply disruptive or emancipatory, but instead trying to take such mediations as necessary and given. At the same time, this presentation offers a critical lens to account for the implications of surveillance and control, which can further disenfranchise queer identities and their access to space/places.

\section{References}

Farman, J. (2012). Mobile Interface Theory: Embodied Space and Locative Media. New York: Routledge.

Shah, N. (2016). Queer mobiles and mobile queers: intersections, vectors, and movements in India. In Hjorth, L. and Khoo, O. (Eds.), Routledge Handbook of New Media in Asia (275-284). Abingdon: Routledge.

McCarthy, A. (2001). Ambient Television: Visual Culture and Public Space. Durham: Duke University press

Nash, C.J. \& Gorman-Murray. (2019). Queer mobilities and new spatial media. In The Geographies of Sexuality. Singapore: Palgrave Macmillan.

Cockayne, D. G and Richardson, L. (2019). The Queer Times of Internet Infrastructure and Digital Systems. In Nash, C.J. \& Murray, A. G. (eds.), The Geographies of Queer Sexualities, 11-28. Singapore: Palgrave Macmillan.

Miles, S. (2017). Sex in the digital city: location-based dating apps and queer urban life. Gender, Place \& Culture, 24(11), 1595-1610. DOI: 10.1080/0966369X.2017.1340874 Safransky, S. (2019). Geographies of algorithmic violence: redlining the smart city.

International Journal of Urban and Regional Research. Retrieved from https://doi.org/10.1111/1468-2427.12833

Shamas, N. (2020, January 23). The many lives of our sexy data bodies. deepdives.in. Retreived from https://deepdives.in/the-many-lives-of-our-sexy-data-bodies$\underline{656 \mathrm{a} 27 \mathrm{fdb} 1 \mathrm{a} 7}$

de Souza e Silva, A. \& Frith, J. (2012). Mobile interfaces in public spaces: Locational privacy, control, and urban sociability. Routledge. 